\title{
Gyral Parcellation of the Cortical Surface Using Geodesic Voronoï Diagrams
}

\author{
A. Cachia ${ }^{1,2,3,4}$, J.-F. Mangin ${ }^{1,2,4}$, D. Rivière ${ }^{1,4}$, \\ D. Papadopoulos-Orfanos ${ }^{1,4}$, I. Bloch ${ }^{3,4}$, and J. Régis ${ }^{5}$ \\ 1 Service Hospitalier Frédéric Joliot, CEA, 91401 Orsay, France \\ mangin@shfj.cea.fr,cachia@shfj.cea.fr, http://www-dsv.cea.fr/ \\ ${ }^{2}$ INSERM ERITM Psychiatrie et Imagerie, Orsay, France \\ 3 Département Traitement du Signal et des Images, CNRS URA 820, ENST, Paris \\ ${ }^{4}$ Institut Fédératif de Recherche 49 (Imagerie Neurofonctionnelle), Paris \\ ${ }^{5}$ Service de Neurochirurgie Fonctionnelle et Stereotaxique, Marseille, France
}

\begin{abstract}
In this paper, we propose a generic automatic approach for the parcellation of the cortical surface into labelled gyri. These gyri are defined from a set of pairs of sulci selected by the user. The selected sulci are first automatically identified in the data, then projected onto the cortical surface. The parcellation stems from two nested Voronoï diagrams computed geodesically to the cortical surface. The first diagram provides the zones of influence of the sulci. The boundary between the two zones of influence of each selected pair of sulcus stands for a gyrus seed. A second diagram yields the gyrus parcellation. The distance underlying the Voronoï diagram allows the method to extrapolate the gyrus limits where the sulci are interrupted. The method is applied on three different brains.
\end{abstract}

\section{Introduction}

The recent advent of automatic methods dedicated to brain morphometry has raised a large interest in the neuroscience community. These tools, indeed, provide a new way of addressing issues related to the links between anatomy and function. While none of these tools can be considered as the perfect one, simply because of the huge complexity of brain anatomy, it is assumed that analyzing hundreds of brains overcome the failures observed for a few ones.

Most of the approaches applied at a large scale rely on a coordinate system, which may be either three dimensional for voxel based morphometry [1], or two dimensional for studies of cortical thickness [814]. In each case, various warping operations are used to match as far as possible the different brains under study with a template endowed with the coordinate system. We will denote this warping principle "iconic spatial normalisation". Morphometry is performed on a point by point statistical basis, either on data related features or on deformation related features [4].

While the coordinate based paradigm has a lot of success in the neuroimaging community, an alternative approach consists of mimicing the classical anatomical morphometry, namely defining some anatomical structures by a segmentation method and deriving some shape descriptors that will be compared across brains. This alternative is sufficiently attractive to be applied manually, although tedious work has to be performed, which prevents large scale studies [22[10]. The motivation behind this "structure based 
morphometry" is the idea that some neuroscience results deeply related to the brain architectural organisation may be either lost during the non perfect iconic spatial normalisation or inaccessible via a coordinate based point of view. Finally, it should be noted that some morphometry approaches are hybrid because local coordinate systems are used to compute some shape descriptors [9].

Two directions of algorithmic research aim at providing automatic methods to perform structure based morphometry. The first one stems directly from the iconic spatial normalisation scheme: a manual segmentation of the template is warped toward any new brain in order to obtain an automatic segmentation [5]. While this approach gives good result for stable brain areas like the deep nuclei, it is more questionable for the cortex [17] because the warping algorithms are disturbed by the high variability of the folding patterns [18,21]. Therefore, a concurent strategy for the cortex consists of linking blind geometric parcellations of the cortex with pattern recognition methods [12,13 21,320], in order to achieve a better definition of sulco-gyral shapes to be compared across brains. A lot of other dedicated segmentation schemes have been defined for various other brain areas [6].

The methods dedicated to the cortex always focus on geometrical properties allowing to devise a definition of cortical folds (depth, curvature, medial axes, etc...). The usual neuroscience point of view about the cortical surface segregation, however, is gyrus based. A gyrus, indeed, is usually considered to be a module of the cortex endowed with dense axonal connexions throughout local white matter [23]. Unfortunately, cortical gyri are relatively difficult to define from a pure geometrical point of view, even if they are supposed to be delimited by two parallel sulci.

In this paper, we propose a two stage strategy for the parcellation of the cortical surface into gyri. First, the main cortical sulci are automatically extracted and identified using a contextual pattern recognition method that may be viewed as a structural alternative to the brain warping approach [21]. Second, the dual gyri are defined as patches of the cortical surface yielded by the computation of two nested Voronoi diagrams, whose initial seeds are inferred from the the identified sulcus bottom lines. This definition of the main gyri is a mixture between geometrical information related to the geodesic distance used to define the diagrams, and a high level cortex model for the recognition of the main sulci which provide the seed lines. The method proposed in this paper, which converts a set of sulci into the set of dual gyri, is generic and may be applied with an alternative different identification of the sulci.

\section{Method}

\subsection{The Sulcus Identification}

The first stage of the method, which has been described by Rivière in [21], provides automatically the set of the main sulci, each sulcus being represented by a set of voxels obtained from a skeleton segmentation (see Fig. 1). For each sulcus, discrete topology properties allows us to obtain the subset of connected voxels corresponding to bottom lines (main part and branches, see Fig.2] [15 16], called sulcal lines. Another outcome of this preprocessing stage is two smooth meshes of the cortex hemispheres endowed with a spherical topology [16]. The sulcus bottoms will be projected on this representation of the cortical surface to define some limits between the dual gyri. Therefore, to have access 
to the method described in this paper, the user has to provide a list of pairs of sulcus names. Each pair will usually correspond to two parallel sulci possibly interrupted.

\subsection{Projection of the Sulcal Bottom Lines onto the Triangulation}

The main problem disturbing the definition of gyri is the interruption of the delimiting sulci, because these interruptions are highly variables. The idea proposed in this paper overcomes this difficulty using the Vorono1 diagram principle. If a set of lines approximatively located at the level of the crowns of the gyri of interest can be provided as gyrus seeds, the whole gyral parcellation can be defined from a distance computed geodesically to the cortical surface. Each gyrus will be the zone of influence of its own seed, namely the subset of the cortical surface closest to its seed than to the other seeds. To try to impose the sulcus bottoms as parts of the boundaries between these influence zones, the idea consists of removing the projected bottom lines from the mesh to prevent the distance to be propagated across these lines. Hence the resulting diagram is inferred from an iterative dilation of the gyrus seeds that is stopped either at the level of the sulcus bottoms, or when two zones of influence get in touch with each other. All the geodesic distance computations used in this paper stem from the thick front propagation idea proposed in [24]. Such distances are also used to apply isotropic geodesic morphological treatments (closing, dilation, etc...)

To make the projected bottom lines behave like walls for the geodesic distance propagation, their connectivity has to be preserved during the projection onto the cortical surface. This is not straightforward because the smooth mesh stems from a decimation algorithm which leads to a non stationary triangle sampling. Morphological closing operations are performed to reach this goal. Another important constraint is the localisation of the projection that has to correspond to the deepest part of the fold on the cortical surface (see Fig. 2). The sequence of processing used to achieve these goals is the following. The $i^{\text {th }}$ Sulcal bottom Line (defined with voxels) is noted $S L_{i}^{v}$; its projection on the triangulation (defined with nodes) is noted $S L_{i}^{t}$. The label of the $i^{\text {th }}$ sulcal line is $l_{i}$. The projection is done for each sulcal line (i.e. for each connected component of the sulcal bottom lines) independently.

1. The first step of the projection is an adaptation of the well-known ICP algorithm [2.7] which allows to find an affine transformation which preserves the global structure of the bottom line. This prevents the creation of large gaps in the middle of the projected points.

- Definition of a set $M A T C H_{i}$ of matched point pairs. The construction is the following: for each point $M_{i}^{v}$ of the sulcal line $S L_{i}^{v}$, a pair $\left(M_{i}^{v}, M_{i}^{t}\right)$ is added to $M A T C H_{i}$, where $M_{i}^{t}$ is the node of the cortical mesh minimising the function $d\left(M_{i}^{v}, M\right)=d_{E}\left(M_{i}^{v}, M\right)+\alpha \operatorname{Depth}(M)$ (see Fig.2), where $\mathrm{M}$ is a mesh node, $\alpha$ ia positive weighting constant, $d_{E}\left(M_{i}^{v}, M\right)$ is the 3D Euclidean distance and $\operatorname{Depth}(M)$ is the geodesic depth. This geodesic depth computation follows the following steps: 1) apply a 3D morphological closing to the white matter binary mask. 2) apply a 3D erosion of $5 \mathrm{~mm}$ to the closed mask. 3) Define all the mesh nodes outside this mask as gyrus crowns. The geodesic depth of all these nodes is then null. 4) Compute the geodesic distance to these crowns (a similar approach may be found in [20]). 
- For each sulcus $i$, perform a least square evaluation of the best affine transformation, which map the sulcus bottom line on the cortical mesh. Each point of $S L_{i}^{v}$ is then projected onto the closest mesh node after affine transformation.

2. The second step consists of closing and thinning the previous projected sulcal lines (see Fig. 3). This operation is applied independently on the projection of each connected component of $S L_{i}^{v}$. The sulcal lines are imposed to be actual lines on the mesh (i.e. a chain of node). The set of projected points is first iteratively dilated geodesically to the triangulation until reaching exactly one connected component. The second stage is a skeletonization like algorithm. The underlying idea is a heuristic computing connected set diameter. A first point is randomly selected in the connected dilated line. A distance (geodesic to the connected set) is computed from this point. The more distant point is selected as the first set extremity. A second (geodesic distance) is computed from this first extremity. The more distant point is selected as the second extremity. The set skeleton (i.e. set diameter) is inferred by a step by step backtracking along the geodesic distance propagation.

\subsection{Gyral Parcellation}

Once the sulci have been projected onto the cortical surface, the remaining processing is embedded into the spherical topology of the cortical surface. The following sequence of stages leads first to the definition of gyral seeds from each pair of sulci given by the user. The second stage leads to the gyral parcellation (see Fig. 3]. The two stages of computation rely on the well known Voronoï diagram notion, which is widely used in computer vision. Such a diagram can be computed into any space domain for a given set of seeds. The diagram is a parcellation of the space into the seed influence zones, where each point is given to the closest seed according to a distance. In the following, this distance is an approximation of the geodesic Euclidean distance [24] (the geodesic distance between two mesh nodes is estimated as the shortest path, through the mesh nodes, linking the two nodes). The diagram is efficiently computed from the previous thick front propagation[24].

A detailed sketch of the process is the following:

1. Computation of the Voronoï diagram of the labelled sulcal lines (see Fig. 3 B) . The nodes that have the label $l_{i}$ correspond to the nodes whose closest seed (i.e. sulcal line), from a geodesic point of view, has the label $l_{i}$. The goal of this diagram is the detection of the boundaries between the zone of influences of the pair of sulci given by the user (see Fig. 3 C). Such a boundary will represent further the seed of the corresponding gyrus. The set of boundaries of the diagram is sometimes called a skeleton by influence zone (SKIZ) [11]. The boundaries are the nodes with at least two different labels in their direct neighborhood. Hence, the boundaries of interest are set of nodes with exactly two labels in their neighborhood corresponding to one of the user specified sulcus pairs. This definition of the gyral seed leads to the ideal localisation for these seeds. The boundary between two neighboring sulcus zones of influence, indeed, is equidistant to the wished gyrus limits. Therefore, during the second diagram computation, the extrapolation of the limits given by the sulcus bottoms will really be equidistant between the crowns of the gyri in competition. 

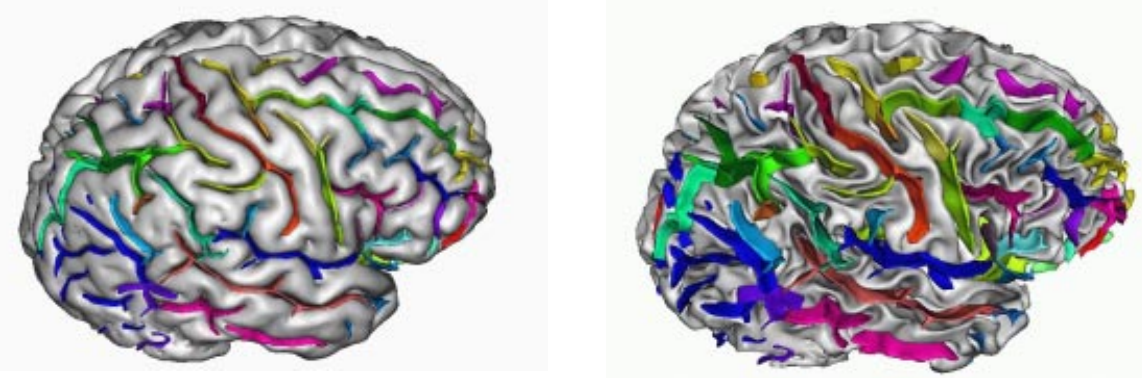

Fig. 1. An example of the result of the sulcus extraction and identification (on right: the white matter mesh used as a spherical model of the cortex). The colors correspond to the various names used by our neuroanatomist to train the recognition system [21]. These names belong to a hierarchy of neuroanatomy names. The sulcus list on which is applied the parcellation is chosen by the system user. Hence several different parcellations can be computed according to the user needs.
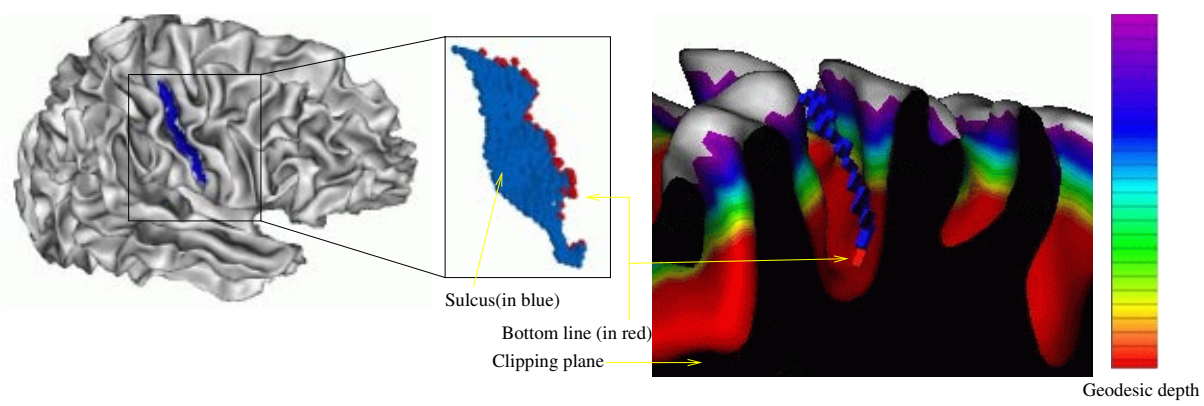

Fig. 2. Each sulcal bottom line is projected onto the cortical surface along the line of maximal geodesic distance to the gyrus crowns.

2. Computation of the second voronoï diagram using the gyrus seeds (see Fig. 3. D). The main difference with the previous diagram is the removal of the sulcal seeds from the mesh, to prevent the distance from crossing the sulcus bottom.

\section{Results and Discussion}

The method has been applied on three different brains. The list of sulcus pairs selected by the user was corresponding to long neighboring parallel sulci, in order to obtain as far as possible the usual anatomical parcellation. While the results provided in Fig. 4 share striking similarities across the three brains and with standard anatomical drawing, some more work has to be done on the sulcus pair selection to reach the more intuitive parcellation.

The huge folding variability highlighted by the figure illustrates the difficulties preventing a pure geometrical definition of gyri. Some frontal sulci that are often long non 

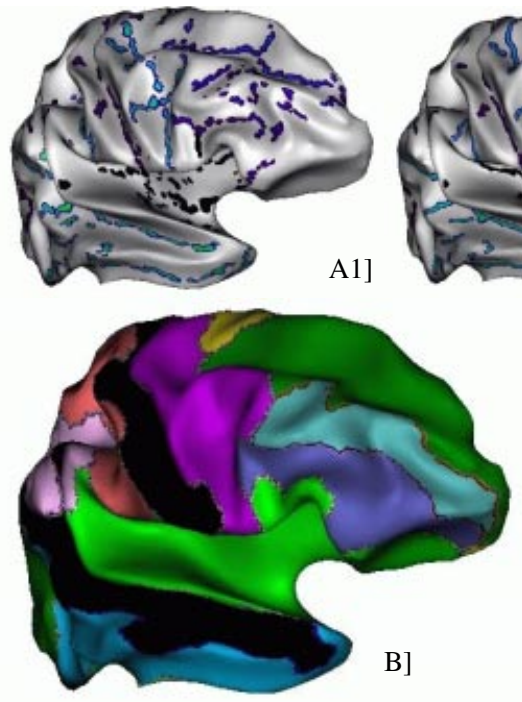

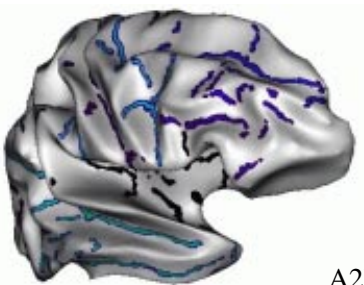

A2]

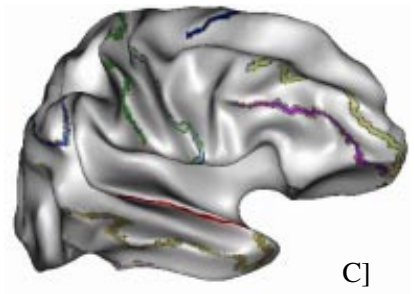

C]

Fig. 3. This figure proposes a sketch of the method mapped on an inflated version of the cortical surface for the sake of understanding. Most of the remaining surface curvature is related to the gyral parcellation targeted by the algorithm. Each sulcus bottom line connected component is first projected (A1). Then the projection is closed using geodesic mathematical morphology, and skeletonised using a geodesic diameter strategy in order to obtain a continuous line (A2). A first Voronoï diagram is computed for the seeds corresponding to these projected lines using a geodesic distance (B). This diagram provides a sulcal based parcellation of the surface. The seeds that will stand for the gyri are boundaries of this first diagram related to the pairs of sulci initially defined by the user (C). Finally a second diagram is computed for these gyral seeds after removal of the sulcal seeds from the mesh in order to prevent the geodesic distance to cross a sulcus bottom (D).
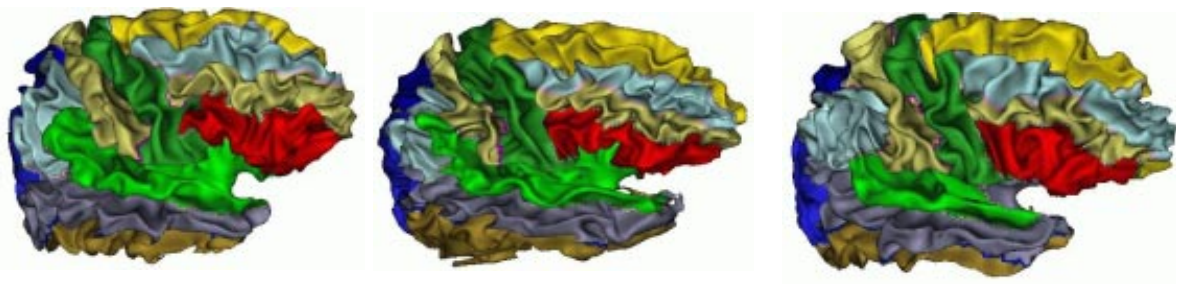

Fig. 4. A typical result obtained from three different brains and some of the main gyri. For instance, the external part of the frontal lobe is split into four parallel horizontal gyri. Back, two vertical gyri correspond to motor and somesthesic areas, etc... Other kind of parcellations can be obtained if the user selects a different list of sulcus pairs.

interrupted furrows can be split into several pieces in some brains. This phenomenum disturbs both the sulcus recognition and the gyrus definition. Nevertheless, our method can extrapolate the standard parcellation to this complex intriguing configurations. Hence, any brain can be processed in a rather consistent automatic way, which opens the door to 
large scale comparisons between pathological and standard subjects. According to the user interest, different sulcus pair list may be provided to the method in order to compare gyral areas and shapes from various definitions.

Another interest of this generic cortical parcellations into gyral patches stems from the recent development of MR diffusion imaging for fiber tracking [19]. The methods used to detect the fiber bundles linking two different cortical areas are still in their infancy, but this new possibility leads now to develop dedicated mapping methods. One possibility is the inference of the matrix of connectivity of the main cortical gyri. For each individual, using gyral patches as input and output may allow the sorting of the huge number of tracked bundles. Then individual matrices of connectivity could be compared on a statistical basis. This approach could provide new research and diagnostic tools for the pathologogies related to the brain connectivity.

\section{References}

1. J. Ashburner and K. J. Friston. Voxel-based morphometry-the methods. NeuroImage, 11:805$821,2000$.

2. P. Besl and N. McKay. A method for registration of $3 \mathrm{~d}$ shapes. IEEE Transaction on Pattern Analysis and Machine Intelligence, 14(2):239-256, Feb 1992.

3. A. Cachia, J.-F. Mangin, D. Rivière, N. Boddaert, A. Andrade, F. Kherif, P. Sonigo, D. Papadopoulos-Orfanos, M. Zilbovicius, J-B. Poline, I. Bloch, F. Brunelle, and J. Régis. A mean curvature based primal sketch to study the cortical folding process from antenatal to adult brain. In Springer Verlag, editor, MICCAI '01, Utrecht, LNCS, pages 897-904, 2001.

4. M. K. Chung, K. J. Worsley, T. Paus, C. Cherif, D. L. Collins, J. N. Giedd, J. L. Rapoport, and A. C. Evans. A unified statistical approach to deformation-based morphometry. NeuroImage, 14(3):595-606, 2001.

5. D. L. Collins, C. J. Holmes, T. M .Peters, and A. C. Evans. Automated 3D model-based neuroanatomical segmentation. Human Brain Mapping, 3:190-208, 1995.

6. J. Duncan and N. Ayache. Medical image analysis: Progress over two decades and the challenges ahead. IEEE Transactions on Pattern Analysis and Machine Intelligence, 22(1):85106, 2000.

7. J. Feldmar and N. Ayache. Rigid, affine and locally affine registration of free-form surfaces. The International Journal of Computer Vision, 18(2), May 1996.

8. B. Fischl, M. I. Sereno, R. B. Tootle, and A. M. Dale. High-resolution intersubject averaging and a coordinate system for the cortical surface. Hum Brain Mapp., 8(4):272-84, 1999.

9. G.Gerig, M Styner, ME Shenton, and JA Lieberman. Shape versus size: Improved understanding of the morphology of brain structures. In MICCAI 2001, LNCS 2208, Springer Verlag, pages 24-32, 2001.

10. Kim J.J., Crespo-Facorro B., Andreasen N.C., O'Leary D.S., Zhang B., Harris G., and Magnotta V.A. An mri-based parcellation method for the temporal lobe. Neuroimage, 11(4):27188, Apr 2000.

11. C. Lantuejoul and S. Beucher. On the use of the geodesic metric in image analysis. J.of Microscopy, 121:39-49, 1981.

12. G. Le Goualher, E. Procyk, D. L. Collins, R. Venugopal, C. Barillot, and A. C. Evans. Automated extraction and variability analysis of sulcal neuroanatomy. IEEE Medical Imaging, 18(3):206-217, 1999.

13. G. Lohmann and D. Y. von Cramon. Automatic labelling of the human cortical surface using sulcal basins. Medical Image analysis, 4(3):179-188, 2000. 
14. D. Mac Donald, N. Kabani, D. Avis, and A. C. Evans. Automated 3-d extraction of inner and outer surfaces of cerebral cortex from mri. Neuroimage, 12(3):340-56, 2000.

15. G. Malandain, G. Bertrand, and N. Ayache. Topological segmentation of discrete surfaces. International Journal of Computer Vision, 10(2):158-183, 1993.

16. J.-F. Mangin, V. Frouin, I. Bloch, J. Regis, and J. López-Krahe. From 3D MR images to structural representations of the cortex topography using topology preserving deformations. J. Mathematical Imaging and Vision, 5(4):297-318, 1995.

17. Tzourio-Mazoyer N., Landeau B., Papathanassiou D., Crivello F., Etard O., Delcroix N., Mazoyer B., and Joliot M. Automated anatomical labeling of activations in spm using a macroscopic anatomical parcellation of the mni mri single-subject brain. Neuroimage, 15(1):273-89, Jan 2002.

18. M. Ono, S. Kubik, and C. D. Abernethey. Atlas of the Cerebral Sulci. Georg Thieme Verlag, 1990.

19. C. Poupon, J.-F. Mangin, C. A. Clark, V. Frouin, J. Régis, D. Le Bihan, and I. Bloch. Towards inference of human brain connectivity from MR diffusion tensor data. Medical Image Analysis, 5:1-15, 2001.

20. M.E. Rettman, Xiao Han, Chenyang Xu, and J.L. Prince. Automated sulcal segmentation using watersheds on the cortical surface. NeuroImage, 15:329-344, 2002.

21. D. Rivière, J.-F. Mangin, D. Papadopoulos, J.-M. Martinez, V. Frouin, and J. Régis. Automatic recognition of cortical sulci using a congregation of neural networks. In MICCAI, Pittsburgh, LNCS-1935, pages 40-49. Springer Verlag, 2000, to appear in Medical Image Analysis.

22. E.R. Sowell, P.M. Thompson, D. Rex, D. Kornsand, K.D. Tessner, T.L. Jernigan, and A.W. Toga. Mapping sulcal pattern asymmetry and local cortical surface gray matter distribution in vivo : maturation in perisylvian cortices. Cerebral Cortex, 12:17-26, Jan 2002.

23. D. C. Van Essen. A tension-based theory of morphogenesis and compact wiring in the central nervous system. Nature, 385:313-318, 1997.

24. B. J. H. Verwer, P. W. Verbeek, and S. T. Dekker. An efficient uniform cost algorithm applied to distance transforms. IEEE PAMI, 11(4):425-428, 1989. 